\title{
Dialectical Behavior Therapy Decreases Depressive Symptoms Among Adolescents in an Acute-Care Inpatient Unit
}

\author{
Ema Saito, MD, ${ }^{1,2}$ Alison A. Tebbett-Mock, $\mathrm{PhD}^{1,2}$ and Madeline McGee, $\mathrm{PhD}^{1,2}$
}

\begin{abstract}
Objective: Research has shown that dialectical behavior therapy (DBT) is an effective treatment modality to decrease suicidal ideation, incidents of nonsuicidal self-injurious behavior, and suicide attempts in an adolescent outpatient population. However, research on the impact of DBT on depressive and manic symptoms for adolescents and within an inpatient setting is limited. The purpose of this study is to examine whether DBT significantly decreases depressive and manic symptoms compared with treatment as usual (TAU) for inpatient adolescents.

Method: We conducted a retrospective chart review for adolescents receiving inpatient DBT and for a historical control group on the same unit before DBT (i.e., TAU). Group differences for continuous outcomes were analyzed using analysis of covariance (ANCOVA), where discharge scores for Hamilton Depression Rating Scale (HAMD), Young Mania Rating Scale (YMRS), Clinical Global Impressions-Severity for Symptoms (CGI-S), and Dynamic Appraisal of Situational Aggression (DASA) were predicted by treatment condition (i.e., DBT and TAU) while co-varying for admission scores and using a $t$ test for CGI-Improvement (CGI-I) in functioning, which was administered upon discharge.

Results: Patients who received DBT had significantly lower HAMD scores, $F(1,409)=5.272, p=0.022, \eta_{\mathrm{p}}{ }^{2}=0.013$, and lower CGI-I scores, $t(596)=2.50, p=0.00$ upon discharge when controlling for admission scores, compared with patients who received TAU. ANCOVAs showed no significant differences on the YMRS, CGI-S, or DASA between the DBT and TAU groups.

Conclusion: DBT on an acute-care inpatient unit for adolescents seems to significantly decrease depressive symptoms during a relatively short period of time compared with TAU. DBT may be an effective treatment modality to decrease depressive symptoms for acute-care inpatient units for adolescents.
\end{abstract}

Keywords: DBT, inpatient, adolescents, depression

\section{Background}

D ALECTICAL BEHAVIOR THERAPY (DBT) is an internationally recognized evidence-based treatment that directly addresses suicidal behavior and other forms of self-injury in adult populations (Linehan 1993). DBT has been adapted for use with adolescents as well and is considered an empirically supported treatment for decreasing repeated suicide attempts (SA) and nonsuicidal self-injury (NSSI) in adolescents (McCauley et al. 2018). However, research on the impact of DBT on depressive and manic symptoms more broadly, as well as domains of functioning, is limited.

The effect of DBT on depressive and anxiety symptoms has been examined for adult patients within inpatient and outpatient settings. Among adult patients with borderline personality disorder (BPD), outpatient DBT treatment has shown efficacy in decreasing depressive and anxiety symptoms, as well as anger (Lynch et al. 2007;
Soler et al. 2009). Outpatient DBT has also been shown to reduce depressive symptoms for adults diagnosed with major depressive disorder (MDD) (Harley et al. 2008; Neacsiu et al. 2014). With an inpatient population, Bohus et al. (2004) reported that adults with BPD who received 3 months of inpatient DBT treatment had significant reductions in depression and anxiety.

There is some support for DBT decreasing mood symptoms within an outpatient adolescent population. Among adolescents treated with DBT, Mehlum et al. (2014) reported that DBT treatment decreased depressive symptoms among those who received 19 weeks of outpatient DBT compared with patients who received enhanced usual care. Goldstein et al. (2015) reported in their pilot study of outpatient DBT treatment for adolescents diagnosed with bipolar disorder that adolescents reported a significant decrease in depressive symptoms over a 1-year treatment course compared with patients who received treatment as usual (TAU).

${ }^{1}$ Division of Child and Adolescent Psychiatry, Department of Psychiatry, Zucker Hillside Hospital, Glen Oaks, New York, USA.

${ }^{2}$ Donald and Barbara Zucker School of Medicine at Hofstra/Northwell, Hempstead, New York, USA.

Funding: No funding was received for this article. 
To date, there is limited research on DBT for adolescents treated within an inpatient setting. Tebbett-Mock et al. (2020) reported in a recently published study that after implementing DBT onto an acute-care psychiatric unit for adolescents with an average length of stay of 8 days, DBT was effective at decreasing incidents of SA, of NSSI, and of restraints, as well as hours of constant observation for self-injurious behavior compared to patients who received TAU before DBT implementation. DBT treated patients also had a shorter length of stay compared with patients who received TAU.

Katz et al. (2004) conducted a feasibility study and compared DBT to TAU on an inpatient unit for adolescents and concluded that the group that received DBT had significantly fewer episodes of violent incidents on the unit compared with the group treated with TAU. McDonell et al. (2010) compared adolescents who received DBT to adolescents who were historical controls and received TAU and found that patients treated with DBT had significantly fewer psychotropic medications prescribed at the time of discharge, fewer incidents of NSSI, and greater improvement in global functioning compared with historical controls. Notably, none of these three studies reported changes in mood symptoms.

The aforementioned findings suggest that DBT may be effective in decreasing mood symptoms, specifically depression among adolescents. Based on the finding that DBT for adolescents in an acute-care inpatient unit was effective in decreasing length of stay and improving safety concerns (i.e., decreased incidents of SA and NSSI and decreased constant observation hours for NSSI), DBT also may be effective in decreasing mood symptoms among an inpatient adolescent population. In this study, we examined the effect of DBT compared with TAU for mood symptoms for adolescents treated on an acute-care inpatient unit.

\section{Methods}

\section{Present study}

This study is a retrospective chart review on adolescents admitted to an acute-care inpatient adolescent unit. The unit is located in a free-standing psychiatric hospital in a suburb of New York City. The present study examines adolescents who were included in a previous study in which the efficacy of DBT was examined compared to TAU among adolescents who required acute-care psychiatric inpatient treatment. The previous study by TebbettMock et al. (2020) examined unit-level variables related to safety (e.g., incidents of SA and NSSI; hours of constant observation; number of restraints and seclusions that occurred on the unit). The current study examines patient-level variables, including clinical rating scales that assess specific symptom severity. We hypothesize that adolescents on an acute-care psychiatric inpatient unit who received DBT will have significantly lower scores on the Hamilton Depression Rating Scale (HAMD; Hamilton 1960), Young Mania Rating Scale (YMRS; Young et al. 1978), Clinical Global Impressions-Improvement (CGI-I), Severity of Symptoms (CGI-S) measures (Guy 1976), and Dynamic Appraisal of Situational Aggression (DASA; Ogloff and Daffern 2006) compared with historical controls who received TAU on the same inpatient unit the year prior.

\section{Participants}

This study includes a total of 801 patients $(n=425$ for DBT group, $n=376$ for TAU group). Patients were aged 12-17 and hospitalized on a coeducational, acute-care inpatient unit. Adolescents were admitted to the inpatient unit either voluntarily or involuntarily through local emergency departments due to imminent safety concerns, including danger to self or others whereby they could not be safely maintained outside of the hospital. DBT patients were those who were hospitalized during an 8-month period of time following implementation of DBT on the unit. Historical controls included all patients who were hospitalized on the same unit during the exact same seasonal span of 8 months the year before DBT implementation. Historical controls received TAU.

\section{Treatment}

The inpatient unit was composed of a multidisciplinary treatment team, including two attending psychiatrists and psychiatry trainees (i.e., residents and child and adolescent fellows), two psychologists and psychology trainees (i.e., externs, interns, fellows), two social workers, two psychiatric rehabilitation specialists, three nurses per shift, and three to four mental health workers per shift.

Details of the DBT treatment and implementation to the unit are available in the previous study (Tebbett-Mock et al. 2020). In short, DBT was implemented on the unit after a multidisciplinary team participated in the DBT Intensive Training by Behavioral Tech, as well as a strategic day of planning with a Behavioral Tech trainer to facilitate treatment adaptations for an acute-care, adolescent psychiatric inpatient unit. DBT included all functions and components of Linehan's original model, as well as adaptations for adolescents made by Miller et al. (2006) (Linehan 1993). Following training, all patients on the unit received DBT milieu treatment, including DBT coaching, a token economy, an egregious behavior protocol requiring chain and solution analyses for egregious behaviors on the unit. All patients received a total of nine DBT skill groups per week, including a 1 hour DBT skill group each weekday targeting skill acquisition (i.e., didactic component) and a 1 hour DBT skill group Tuesday to Friday targeting skill strengthening and generalization (i.e., homework review, behavioral practice, and role plays). Groups included all five adolescent DBT modules: Mindfulness, Distress Tolerance, Emotion Regulation, Interpersonal Effectiveness, and Middle Path (Miller et al. 2006).

All patients received intensive psychotherapy, including $\sim 3$ individual sessions per week and 1-2 family/collateral therapy sessions per week provided by psychology, psychiatry, and/or social work staff. Approximately half of the patients on the unit at any given time were assigned to DBT individual and family therapy provided by psychology staff, and the other half of the patients received either Cognitive Behavioral Therapy (CBT) or supportive psychotherapy provided by social work or psychiatry staff. Assignment of individual therapists was decided based upon DBT therapist availability at time of admission. Nine multidisciplinary team members who participated in DBT training by Behavioral Tech met weekly for consultation team. Consultation team members provided training, supervision, and didactic series to staff across the three work shifts on the unit to facilitate implementation of and fidelity to DBT.

Historical control patients on the unit received TAU. TAU consisted of milieu treatment composed of a token economy system, 3-4 CBT skill groups per week conducted by psychology staff, 10 activity groups per week focused on general coping skills and mental health wellness led by psychiatric rehabilitation specialists, and intensive psychotherapy. Approximately three individual sessions per week and one to two family/collateral therapy sessions per week were provided by psychology or psychiatry staff, and treatment provided was CBT, family systems, psychoeducational, and/or supportive in nature. 
There were several similarities in the treatments received by both DBT and TAU patients. First, all patients received medication management, if deemed necessary. Second, all patients received case management and discharge planning. Third, all staff were trained by the hospital in de-escalation and crisis management skills as part of the hospital's policy and procedure upon staff hiring. Fourth, the same nine primary multidisciplinary team members provided treatment for both DBT and TAU groups. Finally, all patients received a special education program through New York City Board of Education.

\section{Procedures}

The health system's Institutional Review Board provided approval for the study. Data were extracted from the archived electronic medical record database by the Quality Management and Information Technology team of the hospital. Data included the following variables: age; gender; race and ethnicity; diagnosis; days hospitalized; and admission and discharge scores for HAMD, YMRS, CGI-I, CGI-S, and DASA.

\section{Variables}

Age, gender, race, and ethnicity were self-reported by the patient at the time of admission. Days hospitalized were calculated based on the day of admission and discharge. These variables were documented in the electronic medical record by the Central Intake department of the health system.

Diagnoses were made by the attending psychiatrists responsible for patient care based upon clinical interviews according to the Diagnostic and Statistical Manual of Mental Disorders, Fifth Edition (DSM-5; American Psychiatric Association 2013) and were documented in the electronic medical record by psychiatrists. HAMD (24 items), YMRS, and CGI were administered by the attending psychiatrists or psychiatry residents or fellows upon admission and discharge, and scores were documented in the electronic medical record by psychiatrists. Patients diagnosed with depressive disorders were administered the HAMD, and patients with bipolar and related disorders were administered the YMRS. Some patients were administered both the HAMD and YMRS upon admission and discharge based on their clinical presentation. Psychiatrists rated all patients on the CGI-S upon admission and discharge and the CGI-I on discharge. For all patients, DASA scores were determined and documented by nurses every evening, starting after the patient's first full day of admission.

The DASA (Ogloff and Daffern 2006) is a tool to assess the likelihood that a patient will become aggressive within a psychiatric inpatient environment in the upcoming 24 hours. The items include impulsivity, unwillingness to follow instructions, sensitive to perceived provocation, easily angered when requests are denied, negative attitudes, and verbal threats, and items are scored 0 if absent or 1 if present. Scores range from 0 to 7 with higher scores indicating higher severity of symptoms and risk.

\section{Missing data}

All measures included in this study have missing data. For the HAMD and YMRS, data are missing for several reasons (i) measures were diagnosis specific, (ii) administration was not feasible upon admission for patients with severe symptoms (e.g., severe psychosis) who could not complete rating scales with psychiatrists, and (iii) as it was an optional clinical tool to be utilized, it was not routinely administered by all psychiatrists on the unit. For the CGI and DASA, data are missing at random.

\section{Data analysis}

Chi square tests and a $t$ test were conducted as preliminary analyses to assess differences between groups (DBT, TAU) on diagnosis, gender, and age. Group differences for continuous outcomes were analyzed using analysis of covariance (ANCOVA), where discharge scores for HAMD, YMRS, CGI-S, and DASA were predicted by treatment condition (i.e., DBT, TAU) while co-varying for admission scores. A $t$ test was conducted to assess differences between groups (DBT, TAU) on CGI-I, which was administered upon discharge.

Levene's test and normality checks were carried out, and the assumptions met for all variables except for CGI-I indicating that equal variances cannot be assumed for the CGI-I and results should be interpreted with caution for this outcome variable.

\section{Results}

A total of 801 patients were included in our analyses. DBT patients included 425 adolescents aged $12-17.92$ years $(M=15.67$, standard deviation $[S D]=1.44)$, and $66.3 \%$ were female patients $(n=282)$. Regarding race, $40.9 \%(n=174)$ identified as White/ Caucasian, $20.2 \%(n=86)$ as Multiracial, $19.8 \%(n=84)$ as African American/Black, 9.9\% $(n=42)$ as Asian, $8.9 \%(n=38)$ as Unknown/ Declined, and $0.2 \%(n=1)$ as Native American/Alaskan. Regarding ethnicity, $74.4 \%(n=319)$ identified as Non-Hispanic/Latino, $13.9 \%(n=59)$ as Hispanic/Latino, and $11.8 \%(n=50)$ as Unknown/ Declined. They had a mean of $\sim 8$ days hospitalized $(M=8.36$, $S D=8.09$ ). Historic control participants who received TAU included 376 adolescents aged $12-17.92$ years $(M=15.59, S D=1.54)$, and $62.7 \%$ were female $(n=236)$. Regarding race, $52.7 \%(n=198)$ identified as White/Caucasian, 22.1\% $(n=83)$ as African American/Black, $12.8 \%(n=48)$ as Multiracial, $8 \%(n=30)$ as Asian, and $4.5 \%(n=17)$ as Unknown/Declined. Regarding ethnicity, $81.9 \%$ $(n=308)$ identified as Non-Hispanic/Latino, 13\% $(n=49)$ as Hispanic/Latino, and 5.1\% $(n=19)$ as Unknown/Declined. They had a mean of $\sim 11$ days hospitalized $(M=10.74, S D=10.57)$. Twentyone patients were excluded from analyses, 16 from DBT and 5 from TAU, due to moderate to severe intellectual disability such that it was not reasonable to consider these patients to have benefited from talk-psychotherapy (i.e., DBT).

Adolescents presented with a range of primary diagnoses, which were collapsed and categorized according to disorder (Table 1). Approximately $80 \%$ of patients had an affective disorder as a primary diagnosis, and $\sim 5 \%$ had a schizophrenia spectrum disorder. Chi square analysis showed an association between treatment type (i.e., DBT and TAU) and diagnosis, $\chi^{2}(10)=18.80, p=0.04$. Post hoc analyses were conducted utilizing a regression-based approach, which yielded $p$-values for each diagnosis. There were significantly more adolescents diagnosed with Anxiety Disorders and Trauma and Stress Related Disorders in the DBT group and with AttentionDeficit/Hyperactivity Disorder in the TAU group; however, these disorders accounted for a relatively small proportion of the total number of patients (see Table 1 for descriptive statistics and $p$-values). There were no statistical differences between DBT and TAU for gender based upon a $\chi^{2}$ analysis, $\chi^{2}(1)=1.12, p=0.29$ or for age based upon a $t$ test, $t(799)=3.46, p=0.45$.

Descriptives for variables are included in Table 2. The percentage of missing data was $48.5 \%(n=389)$ for HAMD, $45 \%$ 
Table 1. Demographics and Primary Diagnoses for Treatment As Usual and Dialectical Behavior THERAPY PATIENTS

\begin{tabular}{|c|c|c|c|c|c|}
\hline & \multicolumn{2}{|c|}{$T A U$} & \multicolumn{2}{|c|}{$D B T$} & \multirow{2}{*}{ p } \\
\hline & $\mathrm{n}$ & $\%$ & $\mathrm{n}$ & $\%$ & \\
\hline \multicolumn{6}{|l|}{ Demographics } \\
\hline Female & 236 & 62.7 & 282 & 66.3 & 0.29 \\
\hline White/Caucasian & 198 & 52.7 & 174 & 40.9 & \\
\hline Multiracial & 48 & 12.8 & 84 & 19.8 & \\
\hline African American & 83 & 22.1 & 42 & 9.9 & \\
\hline Asian & 30 & 8.0 & 38 & 8.9 & \\
\hline \multicolumn{6}{|l|}{ Diagnosis } \\
\hline ADHD & 9 & 2.4 & 3 & 0.7 & $0.05^{*}$ \\
\hline Anxiety disorder & 3 & 0.8 & 11 & 2.6 & $0.05^{*}$ \\
\hline Bipolar and related disorder & 137 & 36.4 & 140 & 32.9 & 0.30 \\
\hline Depressive disorder & 177 & 47.1 & 197 & 46.4 & 0.84 \\
\hline $\begin{array}{l}\text { Disruptive, impulse control, } \\
\text { conduct }\end{array}$ & 16 & 4.3 & 11 & 2.6 & 0.19 \\
\hline Eating disorder & 0 & 0 & 2 & 0.5 & 0.18 \\
\hline Obsessive compulsive disorder & 3 & 0.8 & 3 & 0.7 & 0.88 \\
\hline $\begin{array}{l}\text { Schizophrenia spectrum } \\
\text { disorder }\end{array}$ & 22 & 5.9 & 33 & 7.8 & 0.29 \\
\hline $\begin{array}{l}\text { Trauma and stress related } \\
\text { disorder }\end{array}$ & 7 & 1.9 & 20 & 4.7 & $0.03 *$ \\
\hline Sleep disorder & 0 & 0 & 1 & 0.2 & 0.35 \\
\hline Substance abuse disorder & 2 & 0.5 & 4 & 0.9 & 0.50 \\
\hline
\end{tabular}

$* p<0.05$.

ADHD, attention-deficit/hyperactivity disorder; DBT, dialectical behavior therapy; TAU, treatment as usual.

$(n=440)$ for YMRS, $25.3 \%(n=203)$ for CGI-I, 9.7\% $(n=78)$ for CGI-S, and $1 \%(n=9)$ for DASA.

There was a significant effect of treatment type on discharge HAMD score after controlling for the effect of admission HAMD score, $F(1,409)=5.272, p=0.022, \eta_{\mathrm{p}}{ }^{2}=0.013$, whereby patients who received DBT had significantly lower discharge HAMD scores compared with patients who received TAU.
According to Cohen's guidelines, the $\eta_{\mathrm{p}}{ }^{2}$ value indicates a small effect size. See Table 3 for ANCOVA results.

Controlling for admission scores, the ANCOVAs showed no significant differences on the YMRS, CGI-S, or DASA discharge scores between patients who received DBT or TAU. See Table 3 for ANCOVA results.

The $t$-test results indicated that patients who received DBT had significantly lower CGI-I scores upon discharge compared with patients who received TAU, $t(596)=2.50, p=0.00$.

\section{Discussion}

Patients who received DBT had significantly lower HAMD scores on discharge compared with patients who received TAU. This result is consistent with previous findings that adolescents treated with outpatient DBT showed significant reductions in depressive symptoms (Mehlum et al. 2014; Goldstein et al. 2015), while extending the findings to an acute-care adolescent inpatient population. Symptomatic improvement also seems to be reflected by significantly lower CGI-I scores among patients who received DBT compared with patients who received TAU.

We acknowledge differences of this study compared with those previously reported. In addition to differences in treatment setting, previous studies are based on a much longer duration of DBT treatment (i.e., 19 weeks to 1 year) compared to our inpatient population with a mean length of stay of 8 and 11 days for DBT and TAU patients, respectively. Although our effect size was small, it is notable that there was a significant reduction in depressive symptoms among our patient population despite a significantly shorter duration of DBT treatment compared with TAU.

Given the comprehensiveness of DBT as a treatment model, including multiple modes of treatment, it is important to consider which mode of the treatment may be the main contributor to improvements in symptoms of depression and severity of symptoms. Emerging literature highlights the impact of DBT skills specifically in effecting change. While it is clear that DBT as a comprehensive treatment model has demonstrated effectiveness in improving depressive symptoms with a BPD population (Bohus et al. 2004; Lynch et al. 2007; Soler et al. 2009) and other mood disorders, such

Table 2. Descriptives for Variables

\begin{tabular}{|c|c|c|c|c|c|c|c|c|c|c|}
\hline \multirow[b]{2}{*}{ Variable } & \multicolumn{5}{|c|}{$T A U$} & \multicolumn{5}{|c|}{$D B T$} \\
\hline & $\mathrm{N}$ & Mean & $S D$ & Median & Range & $\mathrm{N}$ & Mean & $S D$ & Median & Range \\
\hline \multicolumn{11}{|l|}{ HAMD } \\
\hline Admission & 226 & 20.72 & 10.515 & 21.00 & $0-42$ & 186 & 21.58 & 11.458 & 21.00 & $0-47$ \\
\hline Discharge & 226 & 12.74 & 10.348 & 10.00 & $0-42$ & 186 & 11.19 & 10.231 & 8.00 & $0-47$ \\
\hline \multicolumn{11}{|l|}{ YMRS } \\
\hline Admission & 184 & 13.40 & 8.438 & 12.50 & $0-38$ & 177 & 13.18 & 9.013 & 11.00 & $0-36$ \\
\hline Discharge & 184 & 7.98 & 7.172 & 6.00 & $0-37$ & 177 & 7.13 & 6.284 & 6.00 & $0-35$ \\
\hline \multicolumn{11}{|c|}{ CGI Improvement } \\
\hline Discharge & 301 & 2.76 & 0.863 & 3.00 & $1-5$ & 297 & 2.60 & 0.711 & 3.00 & $1-4$ \\
\hline \multicolumn{11}{|c|}{ CGI Severity for Sypmtoms } \\
\hline Admission & 350 & 5.11 & 1.035 & 5.00 & $3-7$ & 373 & 5.04 & 0.951 & 5.00 & $1-7$ \\
\hline Discharge & 350 & 3.96 & 1.013 & 5.00 & $1-7$ & 373 & 3.89 & 1.095 & 4.00 & $1-7$ \\
\hline \multicolumn{11}{|l|}{ DASA } \\
\hline Admission & 369 & 0.488 & 1.275 & 0.00 & $0-7$ & 423 & 0.645 & 1.391 & 0.00 & $0-7$ \\
\hline Discharge & 369 & 0.526 & 1.333 & 0.00 & $0-7$ & 423 & 0.589 & 1.370 & 0.00 & $0-7$ \\
\hline
\end{tabular}

HAMD, Hamilton Depression Rating Scale; YMRS, Young Mania Rating Scale; CGI, Clinical Global Impressions; DASA, Dynamic Appraisal of Situational Aggression; SD, standard deviation. 
Table 3. Analysis of Covariance Results

\begin{tabular}{lccrcccc}
\hline $\begin{array}{l}\text { Dependent } \\
\text { variable }\end{array}$ & $\begin{array}{c}\text { Type III sum } \\
\text { of squares }\end{array}$ & df & $\begin{array}{c}\text { Mean } \\
\text { square }\end{array}$ & $\mathrm{F}$ & $\mathrm{p}$ & $\eta_{p}{ }^{2}$ \\
\hline HAMD & 398.921 & 1 & 398.921 & 5.272 & $0.022^{*}$ & 0.013 \\
YMRS & 50.798 & 1 & 50.798 & 1.650 & 0.200 & 0.005 \\
CGI-S & 0.287 & 1 & 0.287 & 0.290 & 0.590 & 0.000 \\
DASA & 0.152 & 1 & 0.152 & 0.087 & 0.768 & 0.000
\end{tabular}

$* p<0.05$.

HAMD, Hamilton Depression Rating Scale; YMRS, Young Mania Rating Scale; CGI-S, Clinical Global Impressions-Severity for Symptoms; DASA, Dynamic Appraisal of Situational Aggression.

as treatment resistant MDD among adults (Harley et al. 2008; Neacsiu et al. 2014), an additional body of literature highlights the pivotal role of DBT skills-only treatment as a primary mechanism of change.

Reductions in depressive symptoms were observed among patients who received only DBT skill group (Lynch et al. 2007; Harley et al. 2008; Soler et al. 2009). In addition, one prior study examining the relationship between DBT skill use and outcome measures indicated that DBT skills use fully mediated the decrease in SA and depression and increase in anger control over time in an outpatient population of women with BPD (Neacsiu et al. 2010).

The DBT skill deficit model proposes that individuals with BPD have deficits in emotion regulation, interpersonal, and distress tolerance skills (Linehan 1993). This theory pertaining to emotion regulation deficits can be reasonably extended to other diagnoses targeted with DBT (e.g., MDD and Bipolar Disorder). The most recently published systematic review and meta-analysis concluded that patients with MDD use maladaptive emotion regulation strategies more often, use adaptive emotion regulation strategies less often, and have lower emotion regulation ability compared with healthy controls (Visted et al. 2018). Thus, improvement in depressive symptoms for patients who received DBT may be attributable to DBT skill group.

Furthermore, mindfulness practice has been shown to improve psychological health by increasing subjective well-being, reducing emotional reactivity, and improving behavioral regulation (Keng et al. 2011). DBT group begins with a mindfulness practice; thus, DBT group on our unit provided ample opportunities, nine times per week, targeting this.

In addition to DBT group, DBT skill use was consistently reinforced by the milieu, including verbal prompting by staff, skill coaching, and the token economy. The TAU group received a general skill focus, primarily by individual therapists, but skill use was not systematically reinforced by entire milieu staff.

Of note, patients in the DBT and TAU groups had equal improvements on the YMRS and CGI-S. Considering the result that DBT patients had significantly shorter length of stay (TebbettMock et al. 2020) compared with TAU patients, DBT patients seemed to improve equally despite and with significantly shorter duration of treatment.

Although DBT targets impulsive behavior and anger, YMRS and DASA scores were not significantly different between patients who received DBT versus TAU. It is possible that the rating scales used in this study might have not been sensitive enough to capture statistically significant changes in these symptoms. It could also be that time was a barrier, such that depressive symptoms may respond quicker to DBT interventions than impulsivity and/or $\sim 8$ days of inpatient treatment is not long enough to observe and target problematic impulsive behaviors.

\section{Limitation of the study}

The results of this study require cautious interpretation. The study is a retrospective chart review using a historical control group, although data were collected prospectively. It is not a direct comparison between DBT versus TAU. Close to half of the patients lacked certain clinical rating scales, which also limits generalizability of the findings.

\section{Conclusion}

In summary, DBT seems to be an effective treatment for an acute-care inpatient unit for adolescents to decrease depressive symptoms compared with TAU in a relatively short time period of 8 days. Adolescents hospitalized in a psychiatric unit represent the most severely disturbed within a clinical population and often exhibit symptoms that may endanger their safety within and outside of the hospital. Given this acuity and the short length of stay, it is notable that DBT was shown to be an effective treatment to reduce acute depressive symptoms and other safety-related outcomes (Tebbett-Mock et al. 2020). These data are based on everyday clinical practice in an academic training setting where care for patients is provided by a multidisciplinary team of clinicians. Due to the nature of the unit with a high overturn of the patient population and rotating trainees, providing treatment to all patients uniformly is often highly challenging. However, the implementation of DBT to the unit provided a consistent and comprehensive treatment model, which appeared to change outcomes for the better. The effect of DBT implementation for an acute-care psychiatric unit for adolescents requires further investigation. Given the nature of inpatient units with high staff and patient turnover, it would be important to examine if these outcomes are maintained as ongoing. It will be clinically relevant to dismantle the modes of DBT that have made the most positive changes in our patient population. The retention of knowledge and actual skill use during and after the hospitalization and at a long-term follow-up may be of specific interest.

\section{Clinical Significance}

DBT on an acute-care inpatient unit for adolescents seems to significantly decrease depressive symptoms during a relatively short period of time compared with TAU. DBT may be an effective treatment modality to decrease depressive symptoms during acutecare inpatient unit stay for adolescents. Further research would benefit adolescents who require acute-care inpatient treatment.

\section{Disclosures}

Dr. E.S. received honorarium from Dainippon Sumitomo Pharmaceutical Company. Drs. A.A.T.-M. and M.M. do not have anything to disclose.

\section{References}

American Psychiatric Association: Diagnostic and Statistical Manual of Mental Disorders, 5th ed. Washington, DC: American Psychiatric Publishing; 2013.

Bohus M, Haaf B, Simms T, Limberger MF, Schmahl C, Unckel C, Lieb K, Linehan M: Effectiveness of inpatient dialectical behavioral therapy for borderline personality disorder: A controlled trial. Behav Res Ther 42:487-499, 2004.

Goldstein TR, Fersch-Podrat RK, Rivera M, Axelson DA, Merranko J, $\mathrm{Yu}$ H, Brent DA, Birmaher B: Dialectical behavior therapy for adolescents with bipolar disorder: Results from a pilot randomized trial. J Child Adolesc Psychopharmacol 25:140-149, 2015. 
Guy W (ed): ECDEU Assessment Manual for Psychopharmacology, Revised Edition. Rockville (Maryland), NIMH Publication, 1976.

Hamilton M: A rating scale for depression. J Neurol Neurosurg Psychiatry 23:56-61, 1960.

Harley R, Sprich S, Safren S, Jacobo M, Fava M: Adaptation of dialectical behavior therapy skills training group for treatmentresistant depression. J Nerv Ment Dis 196:136-143, 2008.

Katz LY, Cox BJ, Gunasekara S, Miller AL: Feasibility of dialectical behavior therapy for suicidal adolescent inpatients. J Am Acad Child Adolesc Psychiatry 43:276-282, 2004.

Keng SL, Smoski MJ, Robins CJ: Effects of mindfulness on psychological health: A review of empirical studies. Clin Psychol Rev 31:1031-1056, 2011.

Linehan MM: Cognitive Behavioral Treatment of Borderline Personality Disorder. New York (New York), Guilford Press, 1993.

Lynch TR, Ceavens JS, Cukrowicz KC, Thorp SR, Bronner L, Beyer $\mathrm{J}$ : Treatment of older adults with co-morbid personality disorder and depression: A dialectical behavior therapy approach. Int $\mathrm{J}$ Geriatr Psychiatry 22:131-143, 2007.

McCauley E, Berk MS, Asarnow, JR, Adrian M, Cohen J, Korslund K, Avina C, Hughes J, Harned M, Gallop R, Linehan MM: Efficacy of dialectical behavior therapy for adolescents at high risk for suicide: A randomized controlled trial. JAMA Psychiatry 75:777$785,2018$.

McDonell MG, Tarantino J, Dubose AP, Matestic A, Steinmetz K, Gailbreath H: A pilot evaluation for dialectical behavioral therapy in adolescent long-term inpatient care. Child Adolesc Ment Health 15:193-196, 2010.

Mehlum L, Tørmoen AJ, Ramberg M, Haga E, Diep LM, Laberg, S, Larsson BS, Stanley BH, Miller AL, Sund AM, Grohølt B: Dialectical behavior therapy for adolescents with repeated suicidal and self-harming behavior: A randomized trial. J Am Acad Child Adolesc Psychiatry 53:1082-1091, 2014.

Miller AL, Rathus JH, Linehan MM: Dialectical Behavior Therapy with Suicidal Adolescents. New York (New York), Gillford Press, 2006.
Neacsiu AD, Eberle JW, Kramer R, Weismann T, Linehan MM: Dialectical behavior therapy skills for transdiagnostic emotion dysregulation: A pilot randomized controlled trial. Behav Res Ther 59:40-51, 2014.

Neacsiu AD, Rizvi SL, Linehan MM: Dialectical behavior therapy skills use as a mediator and outcome of treatment for borderline personality disorder. Behav Res Ther 48:832-839, 2010.

Ogloff JR, Daffern M: The dynamic appraisal of situational aggression: An instrument to assess risk for imminent aggression in psychiatric inpatients. Behav Sci Law 24:799-813, 2006.

Soler J, Pascual JC, Tiana T, Cebrià, Barrachina, J, Campins MJ, Gich I, Alvarez E, Pérez V: Dialectical behaviour therapy skills training compared to standard group therapy in borderline personality disorder: A 3-month randomized controlled clinical trial. Behav Res Ther 47:353-358, 2009.

Tebbett-Mock AA, Saito E, McGee M, Woloszyn P, Venuti M: Dialectical behavior therapy versus treatment as usual for acutecare inpatient adolescents. J Am Acad Child Adolesec Psychiatry 59:149-156, 2020

Visted E, Volledtad J, Nielsen MB, Schanche E. Emotion regulation in current and remitted depression: A systematic review and metaanalysis. Front Psychol 9:756, 2018.

Young RC, Biggs JT, Ziegler VE, Meyer DA: A rating scale for mania, reliability, validity and sensitivity. Br J Psychiatry 133:429435, 1978.

Address correspondence to: Ema Saito, MD

Division of Child and Adolescent Psychiatry Department of Psychiatry Zucker Hillside Hospital 1 West Behavioral Health Pavilion 75-59 263rd Street Glen Oaks, NY 11004 USA

E-mail: esaito@northwell.edu 